\title{
Demand-Side Management for Regulation Service Provisioning through Internal Pricing *
}

\author{
Ioannis Ch. Paschalidis, ${ }^{\dagger}$ Senior Member, IEEE, Binbin Li, ${ }^{\ddagger}$ Student Member, IEEE, \\ and Michael C. Caramanis, ${ }^{\S}$ Member, IEEE
}

\begin{abstract}
We develop a market-based mechanism that enables a building Smart Microgrid Operator (SMO) to offer regulation service reserves and meet the associated obligation of fast response to commands issued by the wholesale market Independent System Operator (ISO) who provides energy and purchases reserves. The proposed market-based mechanism allows the SMO to control the behavior of internal loads through price signals and to provide feedback to the ISO. A regulation service reserves quantity is transacted between the SMO and the ISO for a relatively long period of time (e.g., a one hour long time-scale). During this period the ISO follows shorter time-scale stochastic dynamics to repeatedly request from the SMO to decrease or increase its consumption. We model the operational task of selecting an optimal short time-scale dynamic pricing policy as a stochastic dynamic program that maximizes average SMO and ISO utility. We then formulate an associated non-linear programming static problem that provides an upper bound on the optimal utility. We study an asymptotic regime in which this upper bound is shown to be tight and the static policy provides an efficient approximation of the dynamic pricing policy. Equally importantly, this framework allows us to optimize the long time-scale decision of determining the optimal regulation service reserve quantity. We demonstrate, verify and validate the proposed approach through a series of Monte Carlo simulations of the controlled system time trajectories.
\end{abstract}

Index Terms-Electricity demand response, electricity regulation service, smart-grid, pricing, electricity markets, welfare maximization, dynamic programming.

\section{INTRODUCTION}

We address advanced demand control in next generation intelligent buildings or neighborhoods that are (i) equipped with a sub-metering and actuation capable smart-microgrid accessible by occupants as well as by a Smart Microgrid Operator (SMO), and (ii) connected to a cyber infrastructure enhanced smart-grid that can support close-to-real-time power market transactions including participants connected at the distribution level. In particular, we consider demand control for offering capacity reserve ancillary services to the Independent

* Research partially supported by the NSF under grants EFRI-0735974 and EFRI-1038230, by the DOE under grant DE-FG52-06NA27490, by the ARO under grant W911NF-11-1-0227, and by the ODDR\&E MURI10 program under grant N00014-10-1-0952.

$\dagger$ I. Ch. Paschalidis is with the Department of Electrical and Computer Engineering and the Division of Systems Engineering, Boston University, 8 Saint Mary's Street, Boston, MA 02215, USA, e-mail: yannisp@bu . edu, url: http://ionia.bu.edu/.

$\ddagger$ B. Li is with the Division of Systems Engineering, Boston University, 15 Saint Mary's Street, Brookline, MA 02446, USA, email: binleon@gmail. com.

$\S$ M. C. Caramanis is with the Department of Mechanical Engineering and the Division of Systems Engineering, Boston University, 15 Saint Mary's Street, Brookline, MA 02446, USA, e-mail: mcaraman@bu.edu.
System Operator (ISO) who clears short-term power markets. In this respect we note that five minute up and down capacity reserves, known as Regulation Service $(R S)$ reserves, are important to meeting the required energy balance and preserving power system stability.

As clean, but alas intermittent and volatile, renewable generation is increasingly integrated into the grid, RS reserve requirements increase as well [1]. Considering that today's RS reserves procured simultaneously with energy correspond to $1 \%$ of load, and command market clearing prices comparable to the price of energy, an increase in $\mathrm{RS}$ requirements without a commensurate increase in supply may well be a show stopper for wind generation expansion. Since centralized generating units are today the only contributor of RS, enabling buildings to offer RS and compete in the power markets promises a major contribution in terms of affordable RS reserve cost and lower $\mathrm{CO}_{2}$ emissions due to the associated adoption of clean generation.

Whole sale power markets were introduced in the US in the mid 1990's [2]. These markets clear simultaneously energy and several types of reserve requirements. For simplicity in exposition we consider here only bidirectional RS reserves. Most markets have not yet allowed the demand side to participate in RS reserves. One of the ISO's, PJM, has allowed loads to participate in energy and reserve transactions since 2006 [3], while other ISO's are contemplating to follow suit. Of the existing short-term markets we point out briefly ([4], [5], [6], [7], [8], [9]) the: (i) day-ahead markets that close at noon of the previous day and clear energy and reserve bids for each of the 24 hours of the next day, (ii) hour-ahead adjustment markets that close an hour in advance and reveal energy and reserve prices, and (iii) 5-minute real-time economic dispatch markets that determine actual ex post variable marginal cost of energy at each bus or node of the transmission system.

We assume that with the advent of the smart grid ([10], [11], [12], [13]) a Load Aggregator (LA) will be able to participate in power markets on a par basis with centralized generators. In particular we assume that an LA will be able to buy energy on an hourly basis at the corresponding clearing price and sell RS reserves for which it will be credited at the system $\mathrm{RS}$ clearing price. An ISO who procures $R_{h} \mathrm{KW}$ of RS is entitled to consider it as a stand-by increment or decrement of consumption that it can utilize at will in total or in part. The ISO may send commands to the RS provider to request that it modulates its consumption either up or down by an amount that does not exceed $R_{h}$. These requests may arrive at interarrival times of 5 seconds or longer [6], [14]. To observe RS 
reserve contractual obligations, the RS provider must deliver the requested increase or decrease in its load with a ramp rate of $R_{h} / 5 \mathrm{KW}$ per minute. The ISO typically re-dispatches the power system in 5 minute intervals. At each 5-minute system dispatch, the ISO economically schedules slower response tertiary reserves so as to reset the utilized RS reserves to their nominal level. As a result, although not guaranteed, the RS reserve provider's tracking of ISO commands is for all practical purposes energy neutral over the long time-scale of an hour and beyond. To meet the aforementioned contractual requirements, the SMO must be capable of controlling loads through the collaboration of a cyber-physical event scheduling layer, the smart microgrid, and a higher decision support and communication layer that interacts with users of energy in order to adapt their demand behaviors to ISO's requests for RS reserve usage. The lower SMO layer consists of sensing and actuation components that collect building state information and actuate so as to safely implement goals determined at the higher level and authorized by building occupants.

This paper focuses expressly on providing the higher decision support layer with a virtual market that operates on the building side of the meter for the purpose of eliciting a collaborative response of building occupants. Our objective is to derive an optimal SMO pricing or incentive policy towards building occupants so that they consent to the sale of RS reserves to the ISO and collaborate in meeting the ISO's RS operational requirements. This objective is in sync with recent calls to develop better mechanisms for reserve management [15]. To the best of our knowledge, little relevant work has been published, and we are the first to propose such a market-based policy for demand control aiming at the provision of RS reserves. Methodologically, related techniques have been used in pricing Internet services [16], [17]. In Sec. II, we detail our internal market-based model and formulate a related welfare maximization problem. In Sec. III we cast the problem into a Dynamic Programming $(D P)$ framework to obtain the optimal dynamic policy conditions. We then proceed to develop performance bounds and approximations. In Sec. IV we develop a static policy and in Sec. V we derive an easily computable upper bound on the optimal performance. Based on this bound, we establish in Sec. VI the asymptotic optimality of the static policy as the load class specific consumption level becomes smaller with a commensurate increase in the number of active loads. Further, we extend the asymptotic optimality results to account for constraints that model energy neutrality over the long time-scale and the upper limit in the RS delivery requested by the ISO. We present numerical results in Sec. VII, and conclude in Sec. VIII.

\section{PROBLEM FORMULATION}

This section models the short time-scale interaction of the SMO with microgrid occupants/loads and the ISO.

The SMO can sell $R_{h} \mathrm{KW}$ of regulation service for the duration of the long time-scale (e.g., one hour), provided that its microgrid's average consumption, $R$, exceeds $R_{h}$ and its consumption capacity is at least $R+R_{h}$. We envision microgrid load classes that can be potentially active during the relevant long time period and include, among others, lights, HVAC zones, computers, electrical appliances and the like. We denote the event of a load unit becoming active as an internal arrival (i.e., internal to the building) and associate a classspecific electricity demand increment with each arrival. We similarly denote the event of a load unit becoming inactive as an internal departure. An actively consuming load unit derives a positive utility. With the sale of $R_{h} \mathrm{KW}$ of $\mathrm{RS}$ the SMO agrees to be on standby and respond to short time-scale (e.g., seconds to minutes) ISO requests for an increment or decrement of the building's consumption. We denote the event of an ISO request as an external arrival (i.e., external to the building). The termination of an ISO request is modeled as an external departure. Note that the cumulative ISO increment or decrement requests can not exceed $R_{h}$ or $-R_{h}$ respectively. As mentioned, the SMO's response does not have to be instantaneous. Most ISOs today, require for regulation service reserves traded in power markets a response rate of roughly $R_{h} / 5 \mathrm{KW}$ per minute. When the ISO request is accommodated by the SMO, the ISO realizes a positive utility whose magnitude depends on system-wide needs and the ISO's obligation to not exceed the maximum amount of $\mathrm{RS}$ it has contracted from the SMO. In addition, in its periodic 5-minute system re-dispatch, the ISO typically attempts to reset its cumulative increment or decrement requests to zero in order to enable RS providers to respond to new requests during future inter-dispatch 5-minute periods. This suggests that the long time-scale average deviation of building consumption from its $R$ level equals zero. Hence, the sale of RS reserves has an energy neutral impact on long time-scale building consumption.

The primary objective is to maximize the sum of SMO and ISO welfare associated with internal and external arrivals. Hard and soft constraints are added to model adherence to the contractual requirements and long time-scale energy neutrality described above. To achieve these goals, the SMO controls the active internal loads and external requests by communicating external and internal-class-specific prices that may be interpreted as dynamic demand control and RS activation feedback signals as much as a monetary exchange.

We assume $M$ classes of internal loads $i=1, \ldots, M$, that arrive according to a Poisson process and require $r_{i}$ $\mathrm{KW}$ for an exponentially distributed period with rate $\mu_{i}$. Let $\boldsymbol{\mu}=\left(\mu_{1}, \ldots, \mu_{M}\right)$ and $\mathbf{r}=\left(r_{1}, \ldots, r_{M}\right)$. Each internal arrival of class $i$ pays an SMO determined price $u_{i}$; we define $\mathbf{u}=\left(u_{1}, \ldots, u_{M}\right)$. We assume that the arrival rate of class $i$ loads is a known demand function $\lambda_{i}\left(u_{i}\right)$ which depends on $u_{i}$ and satisfies Assumption A below. We denote the number of active class $i$ internal loads at time $t$ by $n_{i}(t), i=1, \ldots, M$, and define $\mathbf{N}(t)=\left(n_{1}(t), \ldots, n_{M}(t)\right)$.

\section{Assumption A}

For every $i$, there exists a price $u_{i, \max }$ beyond which the demand $\lambda_{i}\left(u_{i}\right)$ becomes zero. Furthermore, the function $\lambda_{i}\left(u_{i}\right)$ is continuous and strictly decreasing in the range $u_{i} \in$ $\left[0, u_{i, \max }\right]$.

ISO requests for the dynamic activation of RS reserves are 
modeled as a special external class. External RS activation requests occur at a rate $a(y)$ where $y$ is an SMO set price and $a(y)$ satisfies Assumption B below. While they are active, external arrivals require $r_{e} \mathrm{KW}$ each. They become inactive upon their departure which follows an exponential distribution with rate $d$. Denoting the number of active external class loads at time $t$ by $m(t)$, the building is being requested by the ISO to consume $R+R_{h}-m(t) r_{e} \mathrm{KW}$. We impose the following two constraints:

$$
\begin{array}{r}
\mathbf{N}(t)^{\prime} \mathbf{r}+m(t) r_{e}=\sum_{i=1}^{M} n_{i}(t) r_{i}+m(t) r_{e} \leq R+R_{h}, \\
m(t) r_{e} \leq 2 R_{h},
\end{array}
$$

where prime denotes transpose. Inequality (1) ensures that at any time $t$ the total capacity usage of all active loads does not exceed the maximal building consumption capacity $R+R_{h}$ even when $m(t)=0$. Inequality (2) ensures that the ISO can not request that the building consumption at time $t$ be decreased below $R-R_{h}$. Fig. 1 depicts the SMO operation and helps consolidate some of our notation.

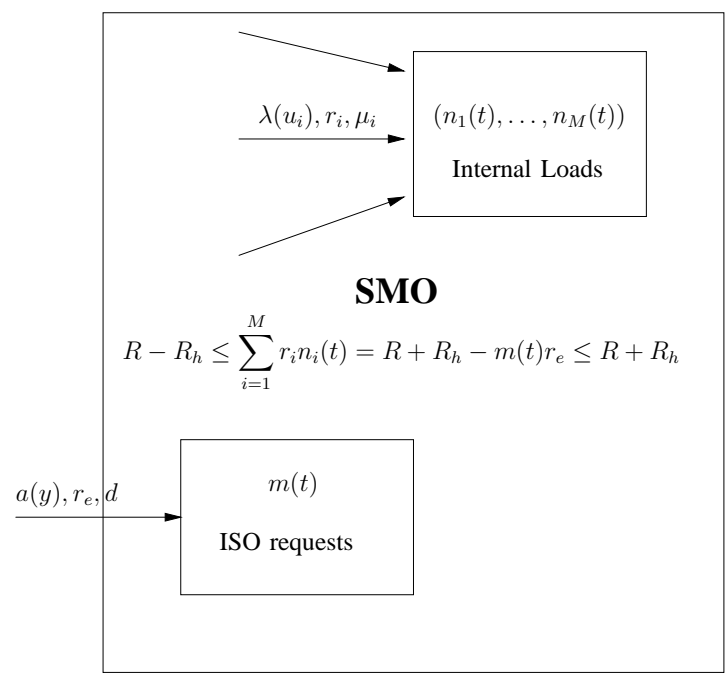

Fig. 1. Schematic representation of controlled internal load and ISO request dynamics. The arrows represent internal (the loads) and external (ISO requests) arrivals characterized by the triplet (arrival rate, load, departure rate).

\section{Assumption B}

There exists a price $y_{\max }$ beyond which the demand $a(y)$ becomes zero. Furthermore, the function $a(y)$ is continuous and strictly decreasing in the range $y \in\left[0, y_{\max }\right]$.

To render the proposed constrained welfare maximization problem more meaningful, we first detail the arrival models and the underlying demand functions. Electricity consumption by an internal load of class $i$ generates utility $U_{i}$, where $U_{i}$ is a non-negative random variable taking values in the range $\left[0, u_{i, \max }\right]$ with a continuous probability density function (pdf) $f_{i}\left(u_{i}\right)$. Arrivals of internal class $i$ loads are seen as being a fraction of potential class $i$ arrivals generated according to a Poisson process with constant rate $\lambda_{i, \max }$. A potential arrival becomes an actual arrival if and only if the random utility realization, $U_{i}$, exceeds the SMO set price $u_{i}$. This implies that internal class $i$ arrivals occur according to a randomly modulated Poisson process with rate $\lambda_{i}\left(u_{i}(t)\right)=\lambda_{i, \max } \mathbb{P}\left[U_{i} \geq u_{i}(t)\right]$. Furthermore, the expected utility conditioned on the fact that a potential arrival has decided to "connect" under a current price of $u_{i}$, is equal to $\mathbb{E}\left[U_{i} \mid U_{i} \geq u_{i}\right]$. We therefore conclude that the expected long-term average rate at which utility accumulates due to internal loads is:

$$
\lim _{T \rightarrow \infty} \frac{1}{T} \sum_{i=1}^{M} \mathbb{E}\left[\int_{0}^{T} \lambda_{i}\left(u_{i}(t)\right) \mathbb{E}\left[U_{i} \mid U_{i} \geq u_{i}(t)\right] d t\right] .
$$

Following a similar argument, the welfare generated from external RS class arrivals can be expressed as:

$$
\lim _{T \rightarrow \infty} \frac{1}{T} \mathbb{E}\left[\int_{0}^{T} a(y(t)) \mathbb{E}[Y \mid Y \geq y(t)] d t\right],
$$

where $Y$ stands for the welfare from the admission of a potential external RS arrival, and $a(y(t))=a_{\max } \mathbb{P}[Y \geq y(t)]$ where $a_{\max }$ is the maximal arrival rate of the external RS class. An interesting interpretation of the long-term average utility generated by external RS class arrivals is that it represents the reservation reward level that the ISO might be willing to pay the SMO for standby RS reserves.

Finally, recall that building response to active ISO RS requests implies that the modified building load must become equal to $R+R_{h}-m(t) r_{e}$ with an allowed adjustment ramp rate of $R_{h} / 5 \mathrm{KW}$ per minute. Although inequality (1) does not allow the building load to ever exceed $R+R_{h}-m(t) r_{e}$, imposing an approximation that is crucial to our asymptotic results, it allows the building load to fall short of $R+R_{h}-m(t) r_{e}$. To impose a reasonable adjustment ramp, and in fact keep it close to the allowed $R_{h} / 5 \mathrm{KW}$ per minute, we impose the following penalty:

$$
\lim _{T \rightarrow \infty} \frac{1}{T} \mathbb{E}\left[\int_{0}^{T} P\left(R+R_{h}-\left(\sum_{i=1}^{M} n_{i}(t) r_{i}+m(t) r_{e}\right)\right) d t\right],
$$

where $P(\cdot)$ denotes the penalty function. We make specific assumptions on the structure of $P(\cdot)$ later.

The optimal pricing policy can now be described as the arg max of:

$$
\begin{aligned}
& \lim _{T \rightarrow \infty} \frac{1}{T} \mathbb{E}\left[\sum_{i=1}^{M} \int_{0}^{T} \lambda_{i}\left(u_{i}(t)\right) \mathbb{E}\left[U_{i} \mid U_{i} \geq u_{i}(t)\right] d t\right. \\
& +\int_{0}^{T} a(y(t)) \mathbb{E}[Y \mid Y \geq y(t)] d t \\
& \left.-\int_{0}^{T} P\left(R+R_{h}-\left(\sum_{i=1}^{M} n_{i}(t) r_{i}+m(t) r_{e}\right)\right) d t\right] .
\end{aligned}
$$

Due to Assumptions $\mathrm{A}$ and $\mathrm{B}$, functions $\lambda_{i}\left(u_{i}\right)$ and $a(y)$ have inverse functions which we denote by $u_{i}\left(\lambda_{i}\right)$ and $y(a)$, respectively. The inverse functions are defined on $\left[0, \lambda_{i, \max }\right]$ and $\left[0, a_{\max }\right]$, respectively, and are continuous and strictly decreasing. This allows us to use the arrival rates $\lambda_{i}$ and $a$ as the SMO's decision variables and write the instantaneous reward rates as $\lambda_{i} \mathbb{E}\left[U_{i} \mid U_{i} \geq u_{i}\left(\lambda_{i}\right)\right]$ and $a \mathbb{E}[Y \mid Y \geq y(a)]$. 
We close this section by recalling that the internal and external (RS) arrivals follow a stochastic process and the utilities associated with these arrivals are random as well. The system responds to these random arrivals by modulating the broadcasted prices in order to maximize the long-term average utility minus the penalty for under-consuming, and thus, not satisfying ISO regulation service requests.

\section{DYNAMIC PROGRAMMING FORMULATION}

The problem introduced in Sec. II is in fact a finite-state, continuous-time, average reward DP problem. Note that the set $\{\mathscr{U}, \mathscr{Y}\}=\left\{(\mathbf{u}, y) \mid 0 \leq u_{i} \leq u_{i, \max }, \forall i ; 0 \leq y \leq y_{\max }\right\}$ of possible price vectors is compact and that all states communicate assuring that there exists a policy that is associated with finite first passage time from any arbitrarily selected state to another state. Standard DP theory results assert that an optimal stationary policy exists.

Since the process $(\mathbf{N}(t), m(t))$ is a continuous-time Markov chain and the total transition rate out of any state is bounded by $\nu=\sum_{i=1}^{M}\left(\lambda_{i, \max }+\mu_{i}\left\lceil\left(R+R_{h}\right) / r_{i}\right\rceil\right)+\left(a_{\max }+d\left\lceil 2 R_{h} / r_{e}\right\rceil\right)$, we can uniformize this Markov chain and derive the following Bellman equation:

$$
\begin{aligned}
& J^{*}=\max _{(\mathbf{u}, y) \in\{\mathscr{U}, \mathscr{Y}\}}\left[\sum_{i \in C(\mathbf{N}, m)} \lambda_{i}\left(u_{i}\right) \mathbb{E}\left[U_{i} \mid U_{i} \geq u_{i}\right]\right. \\
& +\mathbf{1}_{D(\mathbf{N}, m)} a(y) \mathbb{E}[Y \mid Y \geq y]-P\left(\left(R+R_{h}\right)-\left(\mathbf{N}^{\prime} \mathbf{r}+m r_{e}\right)\right) \\
& +\sum_{i \in C(\mathbf{N}, m)} \lambda_{i}\left(u_{i}\right)\left(h\left(\mathbf{N}+\mathbf{e}_{i}, m\right)-h(\mathbf{N}, m)\right) \\
& +\sum_{i=1}^{M} n_{i} \mu_{i}\left(h\left(\mathbf{N}-\mathbf{e}_{i}, m\right)-h(\mathbf{N}, m)\right) \\
& +\mathbf{1}_{D(\mathbf{N}, m)} a(y)(h(\mathbf{N}, m+1)-h(\mathbf{N}, m)) \\
& +m d(h(\mathbf{N}, m-1)-h(\mathbf{N}, m))] .
\end{aligned}
$$

Here, the scalar $J^{*}$ stands for the optimal expected social welfare per unit of time and $h(\mathbf{N}, m)$ denotes the relative reward in state $(\mathbf{N}, m)$. Furthermore, $C(\mathbf{N}, m)=\{i \mid(\mathbf{N}+$ $\left.\left.\mathbf{e}_{i}\right)^{\prime} \mathbf{r}+m r_{e} \leq R+R_{h}\right\}$ is the set of internal class arrivals that can be admitted in state $(\mathbf{N}, m)$, and $D(\mathbf{N}, m)=\left\{\mathbf{N}^{\prime} \mathbf{r}+\right.$ $(m+1) r_{e} \leq R+R_{h}$ while $\left.(m+1) r_{e} \leq 2 R_{h}\right\}$ describe the conditions under which external RS class arrivals can be admitted to the system. We use $\mathbf{1}_{C(\mathbf{N}, m)}$ to denote the indicator function of $C(\mathbf{N}, m)$, i.e., the function equal to 1 if $(\mathbf{N}, m) \in C(\mathbf{N}, m)$ and 0 otherwise. Similarly, $\mathbf{1}_{D(\mathbf{N}, m)}$ denotes the indicator function of $D(\mathbf{N}, m)$.

The above Bellman equation has a unique solution $J^{*}$ and $h(\cdot)$ for an arbitrarily selected special state, say 0 at which we specify the value of the differential cost function, for example $h(\mathbf{0})=0$. Solving Bellman's equation yields an optimal policy that maps any state $(\mathbf{N}, m)$ to the optimal price vector $(\mathbf{u}, y)$ that maximizes the right-hand side of Equation (4). Unfortunately, the curse of dimensionality stipulates that Bellman's equation is only solvable for a small state space. We therefore seek a near-optimal solution that is applicable to SMO's managing relatively large buildings or neighborhoods with a large population of internal loads.

\section{STATIC PRICING POLICY}

We consider a static pricing policy, namely a fixed price vector $(\mathbf{u}, y)$ independent of the system state, for two reasons: (1) the computational effort for obtaining optimal dynamic prices increases exponentially in the number of classes and active loads, and (2) good static prices can be constructed tractably and under reasonable conditions lead to reasonably behaved provisioning of RS. Indeed, under a static pricing policy $(\mathbf{u}, y)$, the system evolves as a continuous-time Markov chain and the corresponding average welfare is given by:

$$
\begin{aligned}
& J(\mathbf{u}, y)=\sum_{i=1}^{M} \lambda_{i}\left(u_{i}\right) \mathbb{E}\left[U_{i} \mid U_{i} \geq u_{i}\right]\left(1-\mathbb{P}_{\text {loss }}^{i}[\mathbf{u}, y]\right) \\
& +a(y) \mathbb{E}[Y \mid Y \geq y]\left(1-\mathbb{Q}_{\text {loss }}[\mathbf{u}, y]\right) \\
& -\mathbb{E}\left[P\left(\left(R+R_{h}\right)-\left(\sum_{i} n_{i} r_{i}+m r_{e}\right)\right)\right]
\end{aligned}
$$

where $\mathbb{P}_{\text {loss }}^{i}[\mathbf{u}, y]$ denotes the steady-state probability $\mathbb{P}\left[\mathbf{N}^{\prime} \mathbf{r}+\right.$ $\left.r_{i}+m r_{e}>R+R_{h}\right]$ that an internal class $i$ arrival is rejected, and $\mathbb{Q}_{\text {loss }}[\mathbf{u}, y]$ denotes the steady-state probability $\mathbb{P}\left[\mathbf{N}^{\prime} \mathbf{r}+\right.$ $(m+1) r_{e}>R+R_{h}$ or $\left.(m+1) r_{e}>2 R_{h}\right]$ that an external RS class arrival is rejected. Moreover, the expected penalty cost is also given by the steady-state probability associated with the same static policy $(\mathbf{u}, y)$.

The optimal static welfare is defined by

$$
J_{s}=\max _{(\mathbf{u}, y) \in\{\mathscr{U}, \mathscr{Y}\}} J(\mathbf{u}, y),
$$

and the following proposition trivially holds.

\section{Proposition IV.1 $J_{s} \leq J^{*}$.}

\section{OPTIMAL PERFORMANCE UPPER BOUND}

In this section we develop an upper bound on $J^{*}$ and use it to quantify the suboptimality of the static policy.

Using the inverse demand functions $u_{i}\left(\lambda_{i}\right)$, and internal class $i$ arrival rate $\lambda_{i}$, the instantaneous reward rate is $F_{i}\left(\lambda_{i}\right)=$ $\lambda_{i} \mathbb{E}\left[U_{i} \mid U_{i} \geq u_{i}\left(\lambda_{i}\right)\right]$. Similarly, $G(y)=a \mathbb{E}[Y \mid Y \geq y(a)]$. Assume that the functions $F_{i}$ and $G$ are concave. Let $J_{\mathbf{u b}}$ be the optimal value of the following Non-Linear Programming $(N L P)$ problem:

$$
\begin{array}{ll}
\max & \sum_{i} F_{i}\left(\lambda_{i}\right)+G(a) \\
& -P\left(R+R_{h}-\left(\sum_{i} n_{i} r_{i}+m r_{e}\right)\right) \\
\text { s.t. } \quad & \lambda_{i}=\mu_{i} n_{i}, \quad \forall i, \\
& a=d m, \\
& \sum_{i} n_{i} r_{i}+m r_{e} \leq R+R_{h}, \\
& m r_{e} \leq 2 R_{h} .
\end{array}
$$

Remark: The non-negativity constraints $n_{i} \geq 0$ and $m \geq 0$ are ignored here. Notice that the departure rates $\mu_{i}$ and $d$ are positive, and the arrival rates $\lambda_{i}$ and $a$ are also non-negative by definition. Thus, $n_{i}$ and $m$ are also non-negative under well-defined demand functions. 
Proposition V.1 If the functions $F_{i}\left(\lambda_{i}\right)$ and $G(a)$ are concave and $P(\cdot)$ is convex, then $J^{*} \leq J_{u b}$.

Proof: Consider an optimal dynamic pricing policy. Without loss of generality, we can assume that the price $u_{i}$ may become large enough to drive the arrival rate $\lambda_{i}\left(u_{i}\right)$ to zero, whenever the state is such that an internal class $i$ arrival cannot be accepted. This amounts to price-directed arrival control. Similarly for the external RS class, $a$ may be driven to zero for sufficiently large $y$. Here, we view $\lambda_{i}, n_{i}$, and $a, m$ as random variables, and use $\mathbb{E}[\cdot]$, to indicate expectation with respect to the steady-state distribution under this particular policy. At any time we have $\sum_{i} n_{i} r_{i}+m r_{e} \leq R+R_{h}$, which implies that $\sum_{i} \mathbb{E}\left[n_{i}\right] r_{i}+\mathbb{E}[m] r_{e} \leq R+R_{h}$. Similarly, we have $\mathbb{E}[m] r_{e} \leq 2 R_{h}$. Furthermore, Little's law implies that $\mathbb{E}\left[\lambda_{i}\right]=\mu_{i} \mathbb{E}\left[n_{i}\right]$, and $\mathbb{E}[a]=d \mathbb{E}[m]$. This shows that the $\mathbb{E}\left[n_{i}\right], \mathbb{E}\left[\lambda_{i}\right], i=1, \ldots, M$, and $\mathbb{E}[m], \mathbb{E}[a]$, form a feasible solution to problem (7). Using the concavity of $F_{i}$ and $G$, the convexity of $P$ and Jensen's inequality

$$
\begin{aligned}
& J_{u b} \geq \sum_{i} F_{i}\left(\mathbb{E}\left[\lambda_{i}\right]\right)+G(\mathbb{E}[a])-P\left(\mathbb{E}\left[R+R_{h}-\left(\sum_{i} n_{i} r_{i}+m r_{e}\right)\right]\right) \\
& \geq \mathbb{E}\left[\sum_{i} F_{i}\left(\lambda_{i}\right)\right]+\mathbb{E}[G(a)] \\
& \quad-\mathbb{E}\left[P\left(R+R_{h}-\left(\sum_{i} n_{i} r_{i}+m r_{e}\right)\right)\right] \\
&=J^{*} .
\end{aligned}
$$

where the last equality is due to the optimality of the policy under consideration.

The optimal solution of NLP (7) provides an upper bound for the optimal social welfare. Moreover, if the objective function of (7) is concave, the NLP is easy to solve.

\section{ASYMPTOTIC BEHAVIOR}

In this section, we consider an asymptotic regime and discuss how to derive the optimal policy while satisfying additional system behavior requirements.

\section{A. Many small loads}

If $R$ and $R_{h}$ are large relative to the required power of a typical arrival, we expect that the laws of large numbers will dominate, attenuate statistical fluctuations, and allow us to carry out an essentially deterministic analysis. To capture a situation of this nature, we start with a base system characterized by finite capacity $R$ and $R_{h}$ and finite demand functions $\lambda_{i}\left(u_{i}\right)$. We then scale the system through a proportional increase of capacity and demand.

More specifically, let $c \geq 1$ be a scaling factor. The scaled system has resources $R^{c}+R_{h}^{c}$, with $R^{c}+R_{h}^{c}=c R+c R_{h}$, and demand functions $\lambda_{i}^{c}\left(u_{i}\right), a_{j}^{c}\left(y_{j}\right)$ given by $\lambda_{i}^{c}\left(u_{i}\right)=c \lambda_{i}\left(u_{i}\right)$ and $a^{c}(y)=c a(y)$. Note that the other parameters $r_{i}, \mu_{i}$, and $r_{e}, d$ are held fixed. We will use a superscript $c$ to denote various quantities of interest in the scaled system.
In this case, consider the NLP problem (7). The upper bound $J_{u b}^{c}$ is obtained by maximizing

$$
\begin{aligned}
\sum_{i} c \lambda_{i}\left(u_{i}\right) \mathbb{E}\left[U_{i} \mid U_{i} \geq u_{i}\right]+c a(y) \mathbb{E}[Y \mid Y \geq y] \\
-P\left(\left(c R+c R_{h}\right)-\left(\sum_{i} \frac{c \lambda_{i}\left(u_{i}\right)}{\mu_{i}} r_{i}+\frac{c a}{d} r_{e}\right)\right),
\end{aligned}
$$

subject to the constraint

$$
\begin{aligned}
\sum_{i} \frac{c \lambda_{i}\left(u_{i}\right)}{\mu_{i}} r_{i}+\frac{c a(y)}{d} r_{e} & \leq c R+c R_{h}, \\
\frac{c a(y)}{d} r_{e} & \leq 2 c R_{h} .
\end{aligned}
$$

It can be seen that, if the penalty function $P(\cdot)$ is linear (cf. Assumption C), then the optimal solution for (7), denoted by $\mathbf{u}_{u b}^{*}=\left(u_{u b, 1}^{*}, \ldots, u_{u b, M}^{*}\right)$ and $y_{u b}^{*}$, is independent of $c$, and $J_{u b}^{c}=c J_{u b}^{1}$. This is stated in Prop. VI.1.

\section{Assumption C}

$P(x)=K x$ for some $K>0$.

Proposition VI.1 Under Assumption C, the optimal objective value of (7) in the scaled system increases linearly with $c$, i.e., $J_{u b}^{c}=c J_{u b}^{1}$.

We are interested in determining the gap between the two bounds derived in Sec. IV and Sec. V. We show that in the regime of many small loads, the following result holds.

Theorem VI.2 Assume that functions $F_{i}\left(\lambda_{i}\right)$ and $G(a)$ are concave, and Assumptions $A, B$, and $C$ hold. Then,

$$
\lim _{c \rightarrow \infty} \frac{1}{c} J_{s}^{c}=\lim _{c \rightarrow \infty} \frac{1}{c} J^{*, c}=\lim _{c \rightarrow c} \frac{1}{c} J_{u b}^{c} .
$$

Proof: It holds from Prop. VI.1 that $J_{u b}^{c}=c J_{u b}^{1}$.

Fix some $\epsilon>0$ and let us consider new static prices $u_{i}^{\epsilon}$ given by $u_{i}^{\epsilon}=u_{u b, i}^{*}+\epsilon$. Let $J^{c}\left(u^{\epsilon}\right)$ be the resulting average welfare. For every $i$ such that $\lambda_{i}\left(u_{u b, i}^{*}\right)>0$, we have $\lambda_{i}\left(u_{i}^{\epsilon}\right)<\lambda_{i}\left(u_{u b, i}^{*}\right)$. Similarly, we set $y^{\epsilon}=y_{u b}^{*}+\epsilon$, and we have $a\left(y^{\epsilon}\right)<a\left(y_{u b}^{*}\right)$. Let $n_{i}^{c}$ (respectively, $n_{i, \infty}^{c}$ ) be the random variable which is equal to the number of active loads of class $i$, in steady-state, in the scaled system, under prices $u_{i}^{\epsilon}$, with capacity $c\left(R+R_{h}\right)$ (respectively, with infinite capacity). Similarly, define $m^{c}$ and $m_{\infty}^{c}$. We obtain

$$
\begin{aligned}
& \mathbb{P}\left[\sum_{i} r_{i} n_{i, \infty}^{c}+r_{e} m_{\infty}^{c}>c R+c R_{h}-r_{\max }\right] \\
\leq & \mathbb{P}\left[\sum_{i} r_{i} n_{i, \infty}^{c}+r_{e} m_{\infty}^{c}>\sum_{i} \frac{c \lambda_{i}\left(u_{u b, i}^{*}\right) r_{i}}{\mu_{i}}+\frac{c a\left(y_{u b}^{*}\right)}{d}-r_{\max }\right] \\
= & \mathbb{P}\left[\sum_{i} r_{i} \frac{n_{i, \infty}^{c}}{c}+r_{e} \frac{m_{\infty}^{c}}{c}>\sum_{i} \frac{\lambda_{i}\left(u_{u b, i}^{*}\right) r_{i}}{\mu_{i}}+\frac{a\left(y_{u b}^{*}\right)}{d}-\frac{r_{\max }}{c}\right],
\end{aligned}
$$

where $r_{\max }=\max \left(\max _{i} r_{i}, r_{e}\right)$ and the 2nd inequality follows from the feasibility of $\mathbf{u}_{u b}^{*}$.

Note that $n_{i, \infty}^{c}$ is equal to the number of customers in an $M / M / \infty$ queue with arrival rate $c \lambda_{i}\left(u_{i}^{\epsilon}\right)$ and service rate 
$\mu_{i}$. As $c \rightarrow \infty$, the random variable $n_{i, \infty}^{c} / c$ converges in probability to $\lambda_{i}\left(u_{i}^{\epsilon}\right) / \mu_{i},{ }^{1}$ which is less than $\lambda_{i}\left(u_{u b, i}^{*}\right) / \mu_{i}$. Similarly, as $c \rightarrow \infty$, the random variable $m_{\infty}^{c} / c$ converges in probability to $a\left(y^{\epsilon}\right) / d$. Therefore, the probability in the right hand side of (10) converges to zero.

Next we consider the RS capacity constraint (2). Similarly,

$$
\begin{aligned}
\mathbb{P}\left[r_{e} m_{\infty}^{c}>2 c R_{h}-r_{e}\right] & \leq \mathbb{P}\left[r_{e} m_{\infty}^{c}>\frac{c a\left(y_{u b}^{*}\right)}{d} r_{e}-r_{e}\right] \\
& =\mathbb{P}\left[\frac{m_{\infty}^{c}}{c}>\frac{a\left(y_{u b}^{*}\right)}{d}-\frac{1}{c}\right],
\end{aligned}
$$

As $c \rightarrow \infty$, by following the same argument as above, the right hand side of (11) converges to zero.

Now compare $n_{i}^{c}$ and $m^{c}$ with $n_{i, \infty}^{c}$ and $m_{\infty}^{c}$, respectively. Comparing the number of active loads in the two corresponding systems (one with capacity $c\left(R+R_{h}\right)$ and the other with infinite capacity), and by defining the arrival processes on a common probability space, we conclude that for all sample paths $n_{i}^{c}$ is smaller than $n_{i, \infty}^{c}$. Similarly, for all sample paths, $m^{c}$ is smaller than $m_{\infty}^{c}$.

Hence,

$$
\begin{aligned}
& \mathbb{P}_{\text {loss }}^{i}\left[\mathbf{u}^{\epsilon}, y^{\epsilon}\right]=\mathbb{P}\left[\sum_{j} r_{j} n_{j}^{c}>c R+c R_{h}-r_{e} m^{c}-r_{i}\right] \\
& \leq \mathbb{P}\left[\sum_{j} r_{j} n_{j}^{c}>c R+c R_{h}-r_{e} m^{c}-r_{\max }\right] \\
& \leq \mathbb{P}\left[\sum_{j} r_{j} n_{j, \infty}^{c}>c R+c R_{h}-r_{e} m_{\infty}^{c}-r_{\max }\right],
\end{aligned}
$$

and the arrival rejection probabilities $P_{\text {loss }}^{i}\left[\mathbf{u}^{\epsilon}, y^{\epsilon}\right]$ converge to zero as well.

$$
\begin{aligned}
\mathbb{Q}_{\text {loss }}\left[\mathbf{u}^{\epsilon}, y^{\epsilon}\right] \leq & \mathbb{P}\left[r_{e} m^{c}>c R+c R_{h}-\sum_{j} r_{j} n_{j}^{c}-r_{e}\right] \\
& +\mathbb{P}\left[r_{e} m^{c}>2 c R_{h}-r_{e}\right] \\
\leq & \mathbb{P}\left[r_{e} m_{\infty}^{c}>c R+c R_{h}-\sum_{j} r_{j} n_{j, \infty}^{c}-r_{\max }\right] \\
& +\mathbb{P}\left[r_{e} m_{\infty}^{c}>2 c R_{h}-r_{e}\right],
\end{aligned}
$$

and the RS class rejection probabilities $\mathbb{Q}_{\text {loss }}\left[\mathbf{u}^{\epsilon}, y^{\epsilon}\right]$ also converge to zero.

By (5) and (6), it follows that

$$
\begin{aligned}
\lim _{c \rightarrow \infty} & \frac{1}{c} J_{s}^{c} \geq \lim _{c \rightarrow \infty} \frac{1}{c} J^{c}\left(\mathbf{u}^{\epsilon}, y^{\epsilon}\right) \\
= & \sum_{i} \lambda_{i}\left(u_{i}^{\epsilon}\right) \mathbb{E}\left[U_{i} \mid U_{i} \geq u_{i}^{\epsilon}\right]+a\left(y^{\epsilon}\right) \mathbb{E}\left[Y \mid Y \geq y^{\epsilon}\right] \\
& -\frac{1}{c} \mathbb{E}\left[P\left(c R+c R_{h}-\left(\sum_{i} n_{i}^{c} r_{i}+m^{c} r_{e}\right)\right)\right] \\
= & \sum_{i} \lambda_{i}\left(u_{i}^{\epsilon}\right) \mathbb{E}\left[U_{i} \mid U_{i} \geq u_{i}^{\epsilon}\right]+a\left(y^{\epsilon}\right) \mathbb{E}\left[Y \mid Y \geq y^{\epsilon}\right]
\end{aligned}
$$

${ }^{1}$ Note that for any $c, n_{i, \infty}^{c}$ has a Poisson distribution with mean $c \lambda_{i}\left(u_{i}^{\epsilon}\right) / \mu_{i}$ and an equal variance. Hence, as $c \rightarrow \infty, n_{i, \infty}^{c} / c$ has a mean equal to $\lambda_{i}\left(u_{i}^{\epsilon}\right) / \mu_{i}$ and a variance that converges to zero.

$$
\begin{aligned}
& -P\left(R+R_{h}-\left(\sum_{i} \frac{1}{c} \mathbb{E}\left[n_{i}^{c}\right] r_{i}+\frac{1}{c} \mathbb{E}\left[m^{c}\right] r_{e}\right)\right) \\
= & \sum_{i} \lambda_{i}\left(u_{i}^{\epsilon}\right) \mathbb{E}\left[U_{i} \mid U_{i} \geq u_{i}^{\epsilon}\right]+a\left(y^{\epsilon}\right) \mathbb{E}\left[Y \mid Y \geq y^{\epsilon}\right] \\
& -P\left(R+R_{h}-\left(\sum_{i} \frac{\lambda_{i}\left(u_{i}^{\epsilon}\right)}{\mu_{i}} r_{i}+\frac{a\left(y^{\epsilon}\right)}{d} r_{e}\right)\right) .
\end{aligned}
$$

This is true for any positive $\epsilon$. We now let $\epsilon$ go to zero, in which case $u_{i}^{\epsilon}$ tends to $u_{u b, i}^{*}$ and $y^{\epsilon}$ tends to $y_{u b}^{*}$. Continuity of the demand function and $P(x)$, imply

$$
\begin{aligned}
& \lim _{c \rightarrow \infty} \frac{1}{c} J_{s}^{c} \\
& \geq \sum_{i} \lambda_{i}\left(u_{u b, i}^{*}\right) \mathbb{E}\left[U_{i} \mid U_{i} \geq u_{u b, i}^{*}\right]+a\left(y_{u b}^{*}\right) \mathbb{E}\left[Y \mid Y \geq y_{u b}^{*}\right] \\
& \quad-P\left(R+R_{h}-\left(\sum_{i} \frac{\lambda_{i}\left(u_{u b, i}^{*}\right)}{\mu_{i}} r_{i}+\frac{a\left(y_{u b}^{*}\right)}{d} r_{e}\right)\right) \\
& =J_{u b}^{1} .
\end{aligned}
$$

Meanwhile, based on Prop. IV.1 and Prop. V.1, $J_{s}^{c} \leq J^{*, c} \leq$ $J_{u b}^{c}=c J_{u b}^{1}$, and the result follows.

In the next subsections, while staying in the regime of many small loads, we explore the structure of the asymptotically optimal policy and consider additional operational constraints and special cases.

\section{B. Energy neutrality}

As discussed in the Introduction, the ISO usually issues RS requests that are related to its balancing area control error and are therefore energy neutral over a longer time-scale (e.g., an hour and beyond) than the one over which the SMO responds to these RS requests.

Mathematically, this means

$$
a \frac{r_{e}}{d}=R_{h} .
$$

This constraint can be added to the NLP in (7) and by solving the resulting problem one obtains a policy that guarantees long-term energy neutrality. Note that (13) implies that the 2nd inequality constraint of problem (7) is inactive. Further, (13) completely specifies the arrival rate of ISO requests (i.e., $a=R_{h} d / r_{e}$ ) and the corresponding price $y(a)$. What is left is to determine the arrival rates of the internal loads $\lambda_{i}$ and the corresponding prices $u_{i}\left(\lambda_{i}\right), i=1, \ldots, M$, which can be done by solving the modified NLP.

\section{Structure of the asymptotically optimal policy}

Let us assume that the energy neutrality constraint (13) is being enforced; the analysis can be extended beyond this case but it is more tedious. Eliminating the two equality constraints of (7), ignoring the 2nd inequality constraint which is inactive due to (13), and dualizing the 1st inequality - capacity constraint, it follows that we need to solve:

$$
\begin{aligned}
\min & -\sum_{i} F_{i}\left(\lambda_{i}\right)-G(a)+K\left(R+R_{h}-\sum_{i} \frac{\lambda_{i}}{\mu_{i}} r_{i}-\frac{a}{d} r_{e}\right) \\
& +q_{1}\left(\sum_{i} \frac{\lambda_{i}}{\mu_{i}} r_{i}+\frac{a}{d} r_{e}-R-R_{h}\right),
\end{aligned}
$$


where $q_{1}$ is the Lagrange multiplier (shadow price) for the capacity constraint.

Therefore, $u_{i}$ should minimize:

$$
-F_{i}\left(\lambda_{i}\left(u_{i}\right)\right)+\left(q_{1}-K\right) \frac{\lambda_{i}\left(u_{i}\right)}{\mu_{i}} r_{i},
$$

where we note that

$$
\begin{gathered}
\lambda_{i}\left(u_{i}\right)=\lambda_{i, \max } \int_{u_{i}}^{u_{i, \max }} f_{i}(v) d v, \\
F_{i}\left(\lambda_{i}\left(u_{i}\right)\right)=\lambda_{i, \max } \int_{u_{i}}^{u_{i, \max }} v f_{i}(v) d v .
\end{gathered}
$$

Setting the derivative of (14) with respect to $u_{i}$ equal to zero and assuming that the utility pdf satisfies $f\left(u_{i}\right)>0$ for all $u_{i} \in\left(0, u_{i, \max }\right)$ it follows that the optimal price $u_{i}^{*}$ for internal load $i$ is provided by the following Proposition.

Proposition VI.3 Under the energy neutrality constraint (13), the optimal price $u_{i}$ for internal load $i$ is given by

$$
u_{i}^{*}=\left[\frac{\left(q_{1}-K\right) r_{i}}{\mu_{i}}\right]^{+}
$$

where $[\cdot]^{+}$denotes projection onto $\left[0, u_{i, \max }\right]$.

We distinguish three cases.

Case 1. If the capacity constraint is inactive, then $q_{1}=0$ by complementary slackness and it follows that $u_{i}^{*}=0$ for all $i$. In this case, the building is consuming less than the ISO requested level and all prices are set to zero to encourage internal arrivals that can increase consumption.

Case 2. Suppose now that the capacity constraint is active but $q_{1}-K<0$, which again yields $u_{i}^{*}=0$ for all $i$. In this case, the cost of poor ISO request tracking, represented by the penalty parameter $K$, is greater than the cost of violating the capacity constraint, which is represented by the Lagrange multiplier $q_{1}$, and prices are again set to zero to encourage internal arrivals and prevent under-consumption.

Case 3. Finally, suppose that the capacity constraint is active but $q_{1}-K>0$. Now the cost of violating the capacity constraint is greater than the cost of under-consuming. It follows that $u_{i}^{*}=\left(q_{1}-K\right) r_{i} / \mu_{i}$, which represents a charge $\left(q_{1}-K\right)$ per unit of "energy" for each internal load $i$. "Energy" here equals the KW power consumed by load $i$ times the expected amount of time that load remains switched on. We note that if $\left(q_{1}-K\right) r_{i} / \mu_{i} \geq u_{i, \max }$ then $u_{i}^{*}$ is capped at $u_{i, \max }$ since there is no reason to increase prices further (the arrival rate drops to zero above $u_{i, \max }$ ).

\section{Linear demand}

Suppose that the internal load utility $U_{i}$ is uniformly distributed in $\left[0, u_{i, \max }\right]$ and the ISO utility $Y$ is also uniformly distributed in $\left[0, y_{\max }\right]$. Then,

$$
\begin{aligned}
& \lambda_{i}\left(u_{i}\right)=\lambda_{i, \max }\left(1-\frac{u_{i}}{u_{i, \max }}\right), a(y)=a_{\max }\left(1-\frac{y}{y_{\max }}\right), \\
& F_{i}\left(\lambda_{i}\right)=u_{i, \max }\left(\lambda_{i}-\frac{\lambda_{i}^{2}}{2 \lambda_{i, \max }}\right),
\end{aligned}
$$

$G(a)=y_{\max }\left(a-\frac{a^{2}}{2 a_{\max }}\right)$.

In this case the NLP (7) becomes a convex Quadratic Programming $(Q P)$ problem for which excellent solvers exist allowing one to solve large instances extremely fast.

\section{E. Optimal selection of $R$ and $R_{h}$}

We have so far considered $R$ and $R_{h}$ values as given market transactions determined at the long time-scale and focused on the operational decisions of the SMO that affect short timescale behavior. We now focus on the SMO's optimal selection of the long time-scale market transactions setting average load $R$ and RS reserves $R_{h}$. We assume that the SMO is a price taker with market conditions reflected in the known ISO utility parameters.

Assuming further that the market energy clearing price is known in advance to the SMO making its RS offer, we let $R_{0}$ be an estimate of the desired building consumption in the event that no RS is offered. Given a feasible setting $\left(R, R_{h}\right)$ with $R \geq R_{0}$ and $R_{h} \geq 0$, we incur two penalty costs: (1) $\frac{1}{2} \kappa_{1}\left\|R-R_{0}\right\|^{2}$ with $\kappa_{1}>0$ to penalize deviation from the desired consumption, and (2) $\frac{1}{2} \kappa_{2} R_{h}$ with $\kappa_{2}>0$ to model the intangible (inconvenience) and tangible (control and actuation) costs of conforming to the short-term response obligations associated with offering regulation service.

Modifying (7) we formulate the following problem:

$$
\begin{array}{ll}
\max & \sum_{i} F_{i}\left(\lambda_{i}\right)+G(a) \\
& -K\left(R+R_{h}-\sum_{i}\left(\lambda_{i} / \mu_{i}\right) r_{i}-(a / d) r_{e}\right) \\
& -\frac{1}{2} \kappa_{1}\left\|R-R_{0}\right\|^{2}-\frac{1}{2} \kappa_{2} R_{h} \\
\text { s.t. } & \sum_{i}\left(\lambda_{i} / \mu_{i}\right) r_{i}+(a / d) r_{e} \leq R+R_{h}, \\
& (a / d) r_{e} \leq 2 R_{h}, \\
& R \geq R_{0}, R_{h} \geq 0,
\end{array}
$$

with decision variables $R, R_{h}$ and the arrival rates $\lambda_{i}$ and $a$. Note that the constraints remain linear and the objective concave, hence, this is a tractable problem. It can be viewed as joint optimization of internal and RS arrival rates (or correspondingly prices) with the building capacity $R$ and the amount of RS quantity offered $R_{h}$.

\section{NUMERICAL EXPERIMENTS}

In this section, we report numerical experiments that verify and validate our results.

Assume that the SMO can support a maximum consumption of $1200 \mathrm{KW}$ with $R=1000 \mathrm{KW}$ and $R_{h}=200 \mathrm{KW}$. This consumption is consistent with the Boston University (BU) Photonics building housing the office of the first author. Consider two internal classes characterized by (all arrival rates are in arrivals/minute and departure rates in departures/minute): $\lambda_{1}\left(u_{1}\right)=1600-80 u_{1}, \lambda_{2}\left(u_{2}\right)=800-80 u_{2}, u_{1, \max }=20$, $u_{2, \max }=10, \lambda_{1, \max }=1600, \lambda_{2, \max }=800, r_{1}=2 \mathrm{KW}$, $r_{2}=1 \mathrm{KW}, \mu_{1}=1, \mu_{2}=2$. The RS class arrival rate is: 
$a(y)=1000\left(1-y / y_{\max }\right)$ with $y_{\max }$ to be determined later, $a_{\max }=1000, r_{e}=1 \mathrm{KW}, d=2$. The penalty function has a slope of $K=1000$. Assume that the social welfare $U_{i}$ is uniformly distributed in $\left[0, u_{i, \max }\right]$ and $Y$ is uniformly distributed in $\left[0, y_{i, \max }\right]$. With these values we can solve the NLP problem (7) (a QP problem) to obtain asymptotically optimal static prices. ${ }^{2}$

We simulate the system for the long time-scale of one hour consisting of 12 periods of 5 minutes each. We consider a scenario according to which one major disturbance and multiple minor disturbances to the system energy balance take place during each 5-minute period. We model the major disturbance by altering the structure of $a(y)$ (changing the value of $\left.y_{\max }\right)$. The minor disturbances are modeled by the random RS class arrivals generated from the Poisson process with rate $a(y)$. We make the reasonable assumption that these disturbances are eventually eliminated over a 15-minute cycle through the 5-minute economic dispatch actions of the ISO who schedules the 15-minute tertiary reserves at its disposal in each 5-minute real-time market clearing.

More specifically in our simulation, a regulation service cycle consists of three 5-minute periods and starts with a full RS standby state, namely, with all RS active loads totalling $R_{h}$. As we mentioned above, this is the result of the ISO 5-minute economic dispatch, which we model by tuning the value of $y_{\max }$. In the following two periods within the cycle, ISO requests - in response to major disturbances - are modeled as random samples from a uniform distribution over $\left[0,2 R_{h}\right]$ instantiated by re-setting the corresponding value of $y_{\max }$. This random cycle is statistically neutral over the long time-scale. In our experiment, $y_{\max }$ changes every 5 minutes and the SMO must control internal class loads to meet ISO requests within the 5-minute requirement of RS reserves. By formulating and solving the NLP problem (7) at the beginning of every period, the SMO is able to appropriately set the prices that result in the required arrivals of internal classes.

The steady-state arrival rates for the two internal classes and the RS class in these periods are shown in Tab. I. The

TABLE I

THE ARRIVAL RATES OF INTERNAL CLASSES AND THE RS CLASS.

\begin{tabular}{llll}
\hline Period & Internal class 1 & Internal class 2 & RS class \\
\hline 1 & 376 & 494 & 400 \\
2 & 409 & 502 & 258 \\
3 & 346 & 486 & 527 \\
4 & 376 & 494 & 400 \\
5 & 309 & 477 & 683 \\
6 & 409 & 502 & 257 \\
7 & 376 & 494 & 400 \\
8 & 322 & 480 & 630 \\
9 & 445 & 511 & 106 \\
10 & 376 & 494 & 400 \\
11 & 403 & 500 & 286 \\
12 & 321 & 480 & 635 \\
\hline
\end{tabular}

evolution of the total consumption due to internal loads and the total load of the RS class are shown in Fig. 2. Note that

${ }^{2}$ We solved the corresponding QP problem using Matlab.

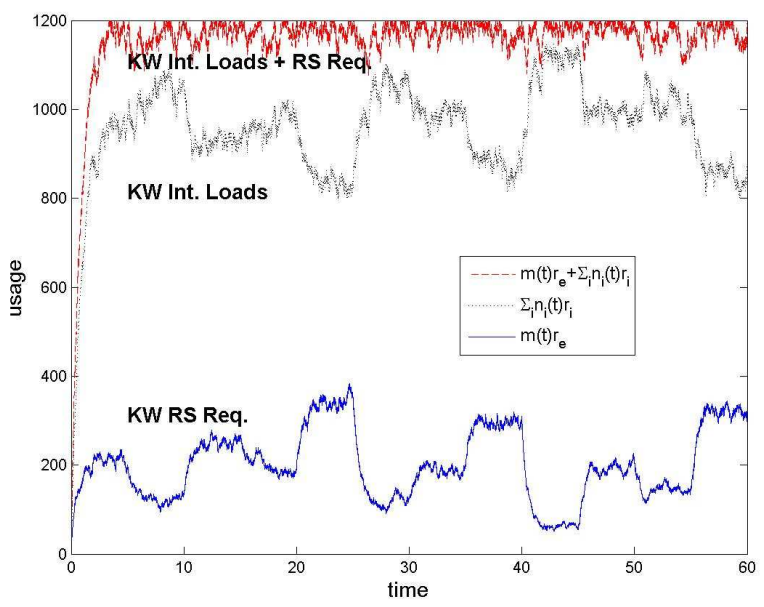

Fig. 2. Energy consumption (in $\mathrm{KW}$ ) by internal classes and active RS requests during the 60 minutes simulated.

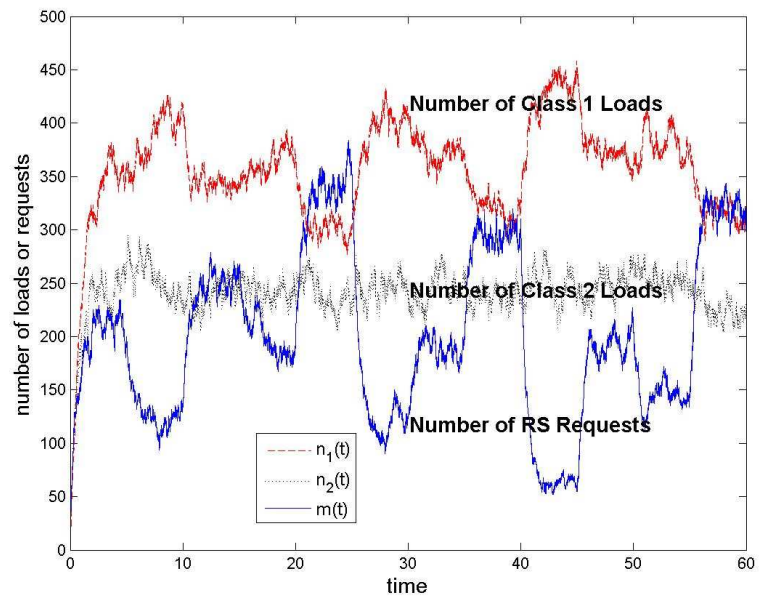

Fig. 3. Number of active internal loads and active RS requests during the 60 minutes simulated.

by applying static pricing policies that are piece-wise constant over each 5-minute period, internal loads converge to the ISO request. Recalling that $\mathrm{RS}$ reserves are required to respond with a ramp of $R_{h} / 5 \mathrm{KW}$ per minute, the response of internal class loads conforms well to requirements. Indeed, since $R_{h}=$ $200 \mathrm{KW}$ in this example, the rate at which $n_{1}(t) r_{1}+n_{2}(t) r_{2}+$ $m(t) r_{e}$ move away from and then approach the $1200 \mathrm{KW}$ level should be close to $40 \mathrm{KW}$ per minute. Figure 2 demonstrates this to be the case. The SMO's decision to offer $200 \mathrm{KW}$ of RS is consistent with its capability to perform according to the associated contractual requirements. In Figure 3, where we plot the number of internal loads and RS requests, we note that there are on average 350 active loads of class 1 with a $2 \mathrm{KW}$ consumption rate - these might be HVAC heating zone loads - and 250 active loads of class 2 with a $1 \mathrm{KW}$ consumption rate. These quantities are consistent with the BU Photonics building which features several hundred heating zones. 


\section{CONCLUSIONS}

The prospect of a paradigm shift in the capabilities of the electric power grid as well as building side-of-the-meter microgrids through Cyber-Physical System (CPS) infrastructure development is within sight. Such CPS infrastructure will certainly enable loads to participate in power markets on a par basis with generating units, not only in the provision of electric energy, but also in the provision of fast reserves. In this paper we develop and test a market-based approach for a Smart Microgrid Operator (SMO) to control numerous and diverse loads and provide such services. We start by formulating a detailed dynamic optimal control problem and then derive an associated tractable and yet near-optimal nonlinear optimization model that is capable of determining both short-term (at the minutes time-scale) operational decision support to the SMO as well as longer time-scale transaction quantities (at the hourly time-scale). Our model is elaborated and validated by numerical simulation results.

A useful insight from our analysis is that as the capacity of the building increases, in conjunction with the number of loads the building can support, static prices (viewed as internal feedback signals) become asymptotically optimal. Such prices are easy to compute and implement. This result suggests that load aggregators that can bundle many buildings have an advantage since they can operate in the limiting regime we analyzed and, as a result, offer static prices to internal loads. We clarify that prices remain static as long as the statistics of load and RS class arrivals, and their corresponding utilities, remain stationary. In practice, we expect these statistics to exhibit time-of-day effects, implying that prices should also change over this very long time-scale. It is remarkable though, that prices need not need to change in the short time-scale of SMO dynamics.

As a final comment, we note that the asymptotic analysis allowed us to forgo the stochastic dynamics and capture the internal market problem in a nonlinear optimization formulation. This enabled the incorporation of a longer-term energy neutrality constraint, as well as, formulating the joint problem of selecting prices together with setting building capacity and the amount of regulation service the building should offer.

\section{REFERENCES}

[1] Y. V. Makarov, C. Loutan, J. Ma, and P. de Mello, "Operational impacts of wind generation on California power systems," IEEE Transactions on Power Systems, vol. 24, no. 2, pp. 1039-1050, 2009.

[2] P. L. Joskow, "Markets for power in the United States: An interim assessment," The Energy Journal, vol. 38, pp. 1-36, 2006.

[3] PJM, White Paper on Integrating Demand and Response into the PJM Ancillary Service Markets, Febuary 2005.

[4] M. Bryson, PJM Manual 12: Balancing Opertions, Revision 16, November 2007.

[5] B. Kranz, R. Pike, and E. Hirst, "Integrated electricity markets in New York," The Electricity Journal, vol. 16, no. 2, pp. 54-65, 2003.

[6] NYISO Day-Ahead Scheduling Manual 11, June 2001.

[7] A. L. Ott, "Experience with PJM market operaton, system design, and implementation," IEEE Transactions on Power Systems, vol. 18, no. 2, pp. 528-534, 2003.

[8] — "Implementation of demand response in the PJM synchronized reserve market," in CIGRE Paris Session and Technical Exhibition, Paris, France, 2008.

[9] PJM, PJM Day-Ahead Scheduling Reserve Market, version 6a, May 2008.
[10] The House of Representatives, "Facilitating the transition to a smart electric grid, May 3, 2007," 2007.

[11] SAIC, "San Diego smart grid study final report," Science Applications International Corporation Smart Grid Team, Tech. Rep. 10-9-06, October 2006.

[12] R. D. Tabors, G. Parker, and M. C. Caramanis, "Development of the smart grid: Missing elements in the policy process," in 43rd Hawaii International Conference on System Sciences (HICSS), January 2010, pp. 1-7.

[13] NIST, NIST Framework and Roadmap for Smart Grid Interoperability Standards, Release 1.0, January 2010.

[14] "NYISO ancillary services manual," available at http://www.nyiso.com/ public/webdocs/documents/manuals/operations/ancserv.pdf, May 2011.

[15] M. Negrete-Pincetic and S. Meyn, "Intelligence by design for the entropic grid," in Power and Energy Society General Meeting, 2011 IEEE, 2011, pp. 1-8.

[16] I. C. Paschalidis and J. N. Tsitsiklis, "Congestion-dependent pricing of network services," IEEE/ACM Trans. Networking, vol. 8, no. 2, pp. $171-184,2000$

[17] I. Paschalidis and Y. Liu, "Pricing in multiservice loss networks: Static pricing, asymptotic optimality, and demand substitution effects," IEEE/ACM Trans. Networking, vol. 10, no. 3, pp. 425-438, 2002.

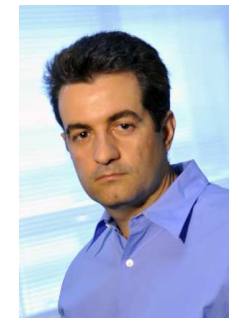

Ioannis Ch. Paschalidis (M'96, SM'06) is a Professor and Distinguished Faculty Fellow at Boston University with appointments in the Department of Electrical and Computer Engineering and the Division of Systems Engineering. He is a Co-Director of the Center for Information and Systems Engineering (CISE) and the Academic Director of the Sensor Network Consortium. He completed his graduate education at the Massachusetts Institute of Technology (MIT) receiving an M.S. (1993) and a Ph.D. (1996), both in Electrical Engineering and Computer Science. In September 1996 he joined Boston University where he has been ever since. He has held visiting appointments with MIT and Columbia University. His current research interests lie in the fields of systems and control, networking, applied probability, optimization, operations research and computational biology.

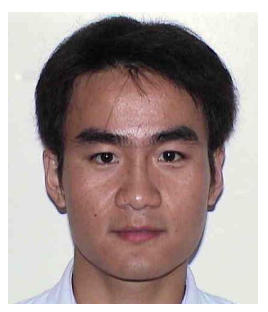

Binbin Li received the B.Sc. degree in automation and the M.Sc. degree in control science and engineering from Tsinghua University, Beijing, China, in 2004 and 2006, respectively. He received a Ph.D. degree in Systems Engineering from Boston University in 2011. His research interests include optimization and decision theory with main applications in communication and sensor networks, and the smartgrid.

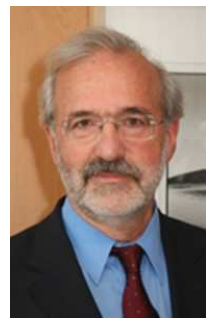

Michael C. Caramanis holds a B.S. (1971) in Chemical Engineering from Stanford University and an M.S. (1972) and Ph.D. (1976) in Engineering with minors in Microeconomics and Economic Development from Harvard University. He is Professor at Boston University with appointments in the Department of Mechanical Engineering and the Division of Systems Engineering. He served at the Greek National Energy council (1976-79), the MIT Energy Laboratory (1979-82), and was chair of the Greek Regulatory Authority for Energy from February 2005 till recently. He researches complex stochastic production systems and decision support with applications in manufacturing systems and real-time electric power markets. 